\title{
COMMITTEE ON RELATIONSHIPS BETWEEN SOLAR AND TERRESTRIAL PHENOMENA
}

On the occasion of the meetings of Commission I2, a joint meeting was held in Cambridge with the Committee on Solar and Terrestrial Relationships. Since the meeting at Leiden the Committee had suffered the heavy loss of two members, Dr L. A. Bauer and General G. Ferrié, whose activity in the Committee had been so great and so highly appreciated. On the termination of the period of office of the Chairman, Prof. Chapman, the International Council of Scientific Unions had nominated Abetti to be Chairman from January 1932.

During the past period Chapman has edited the Third Report, which contains much valuable information on the work done by members of the Committee and other investigators, either on solar research or on terrestrial magnetism, or on the relations between Sun and Earth by means of wireless, ultra-violet radiation, aurorae, etc.

The work is various and important, but we must admit that little advance has been made in the explanation of solar and terrestrial correlations. To sum up the situation the following remarks have been put together by Chapman and have been presented to the joint meeting by Abetti. These remarks become specially important if compared with the report presented by Brunner in another meeting of Commission I2:

\section{Some Remarks on Solar and Terrestrial Relationships}

\section{By S. ChapMAN}

Whereas the general solar radiation is variable only within narrow limits, the phenomena of terrestrial magnetism give evidence of considerable variations in components of the solar radiations, either ultra-violet light or corpuscles, that are absorbed at great heights in the earth's atmosphere. Some of these phenomena, at least, are connected with the ionization of the upper atmosphere, which can now be investigated, rather directly, by radio methods. The latter give the height and maximum electron density over a particular region at the time of measurement, while the magnetic observations give more general information about the state of ionization over large parts of the globe, and have the advantage of being continuously recorded, with accumulated data extending backwards over many decades.

The magnetic variations can be analysed into different components, which possess different relationships to the sun. The irregular magnetic disturbance, known as magnetic storms when specially intense, varies in intensity in rough parallelism with the sunspot cycle; it also shows a 27 -day recurrence tendency plainly connected with the solar rotation, though this tendency is apparent only in the weaker disturbance. The great magnetic storms do not show it*, but on the other hand they show a rather close relationship with striking sunspots, which is not perceptible in the case of weak disturbance.

In order to investigate further the relation between magnetic disturbance and solar conditions the International Astronomical Union has instituted its Bulletins

\footnotetext{
* Greaves and Newton, M.N.R.A.S. 89, 84, 64I.
} 
of daily solar character figures partly for comparison with the magnetic character figures which have long been available. In a valuable paper shortly to be published in the journal Terrestrial Magnetism, J. Bartels has studied this material, with results that may disappoint some of the hopes entertained when the Solar Bulletins were instituted, but which are an important outcome of those Bulletins. He finds a high degree of correlation between the various solar character figures inter se, and a low correlation between any of them and the magnetic character figures. He has also made two diagrams*, one for daily sunspot numbers, and one for daily magnetic character figures, somewhat similar to a diagram (for magnetic storms) published many years ago by Maunder, in which data for each day of each solar rotation are represented along a horizontal row, successive rotations being represented by successive rows. Both of these diagrams show the 27 -day recurrence tendency very prominently, but the sequences for the sunspot numbers and for magnetic activity seem to have little relation with one another. The inference is that the ordinary low-intensity magnetic activity is not closely connected with sunspots nor (in view of the close correlation between these and the other solar character figures) with the other phenomena to which the Solar Bulletins refer. This is not an indication that the latter have failed in their purpose, which indeed is partly achieved even by this negative result; doubtless, moreover, as in the case of the magnetic character figures, further important uses for the figures in the Solar Bulletins will gradually appear, and it is very desirable that the Bulletins should be continued over a sufficiently long period (at least two sunspot cycles) for statistical discussion. But Bartels' results do suggest that the solar influences that determine the degree of terrestrial magnetic disturbance are not visually prominent on the sun, and have as yet escaped detection. It may be hoped that the more continuous and intensive observation permitted by the spectrohelioscope will lead to their discovery. Beyond this it is not clear what should be looked for that has not yet been investigated, except that a close examination of the solar spectrum at times when the earth is actually experiencing a great magnetic storm may give results of value $\uparrow$.

In addition to magnetic disturbance, the solar diurnal variation of terrestrial magnetism undergoes notable changes of intensity, partly in parallel with the sunspot cycle and also irregularly from day to day, even in a period of extreme magnetic calm $\ddagger$. These are presumably associated with changes in the ionization of the upper atmosphere; radio studies of this ionization give promise of checking this conclusion, and of indicating which of the two ionized layers is associated with this diurnal magnetic variation. These studies, which must be chiefly pursued by investigation of the geophysical data by magnetic and radio methods, should lead to information as to solar radiations, not improbably in the ultra-violet spectrum, which are inaccessible to astrophysical observation.

In this connection, mention may be made of the radio observations to be made during the eclipse of August I932, in order to test whether either of the two ionized layers is ionized by neutral corpuscles (as I have elsewhere suggested on magnetic grounds§). On the latter hypothesis the moon would eclipse the stream

* Shown at the recent Exhibition of the Department of Terrestrial Magnetism of the Carnegie Institute of Washington.

† S. Chapman, M.N.R.A.S. 89, 466, r929.

† S. Chapman and J. M. Stagg, Proc. Roy. Soc. Lond. A, 123, 27, 1929; and 130, 668, 1931; also J. Bartels, Trans. Amer. Geophys. Union, I931, p. 126.

$\S$ Proc. Roy. Soc. Lond. A, 132, 353, $193 \mathbf{1}$. 
of corpuscles impinging on the earth two hours before the optical eclipse occurs (the interval of time depending on the assumed velocity of the particles); the "corpuscular" eclipse track will also be correspondingly to the east of the optical eclipse track. These consequences of the hypothesis of corpuscular ionization of the (lower) ionized layer in the earth's atmosphere should be decisively confirmed or disproved at an early date, through the radio eclipse observations*.

One further eclipse observation that might throw valuable light on the sun's influence upon the upper atmosphere is the examination of the green light $\left(\lambda_{5577}\right)$ of the non-polar aurora, which should be visible in the sky during the period of total eclipse. A rough photometric estimation of its intensity, for comparison with the intensity observed in the night sky $\dagger$, would be of considerable theoretical interest, particularly in view of the probability that the intensity of this light varies in parallel with sunspot cycle.

Attention may be drawn to the recent issue of the Third Report of the Commission appointed by the International Research Council to further the study of Solar and Terrestrial Relationships. Perhaps the most important advances there reported upon relate to solar influences upon radiotelegraphy.

Fleming also points, by correspondence, to the work of Bartels and to the following conclusion:

"There must exist on the sun's surface certain restricted areas-which we have ventured to designate M-regions-the life of which is limited usually to not more than a year though generally longer than that of sunspots. They cause terrestrialmagnetic disturbances probably by emitting well-defined corpuscular streams. Such solar regions in the individual sense escape the usual astrophysical method of observation (visual, photographic, and spectrohelioscopic) and can as yet be traced only in terrestrial-magnetic activity. Terrestrial-magnetic records have obtained a purely astrophysical interest beyond their well-known geophysical aspect, namely, the indication of the times when the Earth is actually under the influence of solar streams."

Future work will be continued on previous lines possibly with increased cooperation in solar observations specially in regard to those to be made with the spectrohelioscope and with investigation of new methods to follow the activity of the sun. Also the improved collaboration to establish better and more complete characteristic figures should prove useful in the question.

To succeed General Ferrié the International Union for Scientific Radiotelegraphy has been asked to nominate its representative. The International Astronomical Union, which had Abetti and St John as its representatives, now nominates d'Azambuja to succeed Abetti.

* M.N.R.A.S. 92, March I932.

$\dagger$ Rayleigh, Proc. Roy. Soc. Lond. A, 129, 458, 1930. 